\title{
Anabases
}

ANABASES Traditions et réceptions de l'Antiquité

$4 \mid 2006$

Varia

\section{Polybe et Montesquieu : aspects d'une réflexion sur le pouvoir}

\section{Marie-Rose Guelfucci}

\section{(2) OpenEdition}

1 Journals

Édition électronique

URL : http://journals.openedition.org/anabases/2893

DOI : 10.4000/anabases.2893

ISSN : 2256-9421

Éditeur

E.R.A.S.M.E.

\section{Édition imprimée}

Date de publication : 1 octobre 2006

Pagination : 125-139

ISSN : 1774-4296

\section{Référence électronique}

Marie-Rose Guelfucci, «Polybe et Montesquieu : aspects d'une réflexion sur le pouvoir », Anabases [En ligne], 4 | 2006, mis en ligne le 01 janvier 2012, consulté le 20 octobre 2019. URL : http:// journals.openedition.org/anabases/2893 ; DOI : 10.4000/anabases.2893 


\section{Polybe et Montesquieu : aspects d'une réflexion sur le pouvoir ${ }^{1}$}

MARIE-Rose GuelfucCI

Si L'IDÉE DE RAPPROCher Montesquieu de Polybe n'est pas une idée nouvelle ${ }^{2}$, elle n’a jamais été véritablement exploitée. Or la lecture que fait Montesquieu de certains éléments fondamentaux des Histoires de Polybe, pour mener en philosophe sa propre réflexion sur le pouvoir romain, conduit à porter sur l'œuvre de l'historien grec un regard différent. Nous nous proposons donc, en nous fondant plus particulièrement sur des rapprochements entre les Histoires et les Considérations sur les causes de la grandeur des Romains et de leur décadence, de préciser dans un premier temps les raisons de ce parallèle. Nous examinerons ensuite, en mettant en relation le texte de Montesquieu avec celui de Polybe, la manière dont l'un et l'autre font apparaître les atouts de Rome dans sa conquête de l'hégémonie, puis les risques courrus par ce pouvoir comme par tout pouvoir établi pour montrer comment, en fin de compte, l'exemple romain dépasse les limites chronologiques dans lesquelles il s'inscrit, pour être, beaucoup plus largement, représentatif d'une philosophie politique et d'une philosophie de l'histoire qui ont, toutes deux aussi, des points communs.

1 Le présent article s'appuie sur une intervention faite dans le cadre d'un séminaire pluridisciplinaire à l'université de Nice Sophia-Antipolis et publiée dans Antiquité et nouveaux Mondes, J. Rieu éd., Nice, 1998, p. 103-131.

2 A. Duraffour, "Les Considérations de Montesquieu dans leurs rapports avec Bossuet et Polybe ", in Mélanges offerts à M. Charles Andler par ses amis et ses élèves, Strasbourg, 1924, p. 129-142. 


\section{Les raisons du parallèle}

La première justification d'un tel parallèle tient, au-delà d'une différence de contenu due au moment historique où ils écrivent l'un et l'autre, à la nature même du sujet qu'ils se proposent : en 168 a.C., le royaume de Macédoine vaincu et la Grèce affaiblie par cette défaite, Polybe veut comprendre, selon la formule qui définit son œuvre, " comment et grâce à quel gouvernement l'État romain a pu... étendre sa domination à presque toute la terre habitée et cela en moins de cinquante-trois ans ${ }^{3}$ ", de 219 à 168. Il consacre donc trente livres de ses Histoires à cette période qui marque la conquête lente et progressive de l'hégémonie par Rome, mais précise ce projet initial dans le véritable prologue de l'œuvre, au début du livre III, et se propose, dans dix livres supplémentaires, de s'interroger sur l'usage que fait Rome de sa victoire une fois sa domination installée, afin que ses lecteurs et les lecteurs à venir puissent imiter ou refuser, en toute connaissance de cause, l'exemple romain. Cette nouvelle œuvre couvre les années 168 à 146 a.C., date à laquelle, pour des raisons et avec des responsabilités différentes, Rome fait détruire Carthage et, après le siège et la destruction partielle de Corinthe, procède au démembrement des confédérations grecques.

Si Polybe est contemporain des faits qui terminent ses Histoires, Montesquieu a, sur les succès et les difficultés de Rome, le recul qui permet d'embrasser la totalité du passé : sur les vingt-trois chapitres de l'œuvre, huit marquent l'ascension romaine depuis les commencements de la cité, le neuvième définit le risque que présente ce pouvoir accru qui, donnant trop d'" autorité au dehors » aux chefs d'armée, accordant le titre de citoyen à trop de peuples divers, perd la république, peu à peu corrompue (X) et, de Sylla à Auguste, conduit Rome à l'empire (X-XIII) ; les chapitres XIV à XXIII montrent la décadence très progressive de l'empire romain. Ce sont donc essentiellement les dix premiers chapitres des Considérations qui, pour le parallèle proposé, nous intéresseront.

Le second point commun entre les deux œuvres n'est pas, si fondamental soit-il, leur travail d'établissement des faits historiques, mais la fin qu'elles poursuivent. Les méthodes sont certes différentes. Polybe rompt avec la conception rhétorique et dramatique de l'histoire qui domine alors et s'explique sur les trois moyens nécessaires à l'historien pour établir la vérité : une expérience politique et militaire personnelle, l'enquête sur le terrain et, en dernier lieu seulement, l'enquête sur des documents (XII. 27-28a). Montesquieu, lui, ne fait pas du tout œuvre d'enquêteur en action, ni même - et on le lui reprochera - œuvre d'antiquaire, mais il ne s'en livre pas moins à un minutieux travail d'information à partir des textes, attesté, quand il réfléchit sur les exemples historiques, par le nombre des notes qui renvoient toujours très précisément aux auteurs

3 Par souci d'unité et pour plus de commodité pour le lecteur, toutes les traductions des Histoires seront, sauf indication contraire, empruntées à Denis Roussel, Polybe Histoire, Gallimard, 1970. 
antiques. Même si l'on ne sait s'il lisait les auteurs grecs dans le texte ou dans une traduction latine, sa documentation est très diverse : Salluste, Tite-Live, Appien, Plutarque, Végèce, pour ne pas citer Polybe, qu'il connaît parfaitement. D'abord publiées à Amsterdam sans nom d'auteur en 1734, avant une présentation à l'Académie française le 30 août, les Considérations reçoivent de la critique contemporaine un accueil sans enthousiasme : Voltaire, dans une lettre à Thiériot de novembre de la même année, en critique le style, déplorant d'avoir affaire à " une table des matières 4 ». Montesquieu reprend plusieurs fois son étude jusqu'à l'édition corrigée dont le texte, presque définitif, paraît en 1748. Après la parution d'une traduction de son travail sur les Romains à Edimbourg en 1751, il donne une réponse très précise aux critiques qui lui sont faites tout en apportant un certain nombre de corrections.

Mais pas plus que Polybe n'est un annaliste attaché à la seule relation suivie des faits, les Considérations ne sont, malgré les mauvais esprits qui, à la parution de l'ouvrage, ironisèrent sur la décadence de Montesquieu, une simple histoire de Rome écrite à très grands traits. En effet, quoique détachées de l'Esprit des Lois, elles en sont une préparation, "histoire de Rome à l'usage des hommes d'État et des philosophes", selon la formule de d'Alembert. Frédéric II annote d'ailleurs le texte et félicite l'auteur. Or c'est aussi aux hommes d'État que Polybe destine son ouvre, en faisant explicitement de l'histoire une leçon.

Les Histoires, en effet, procèdent d'abord de l'intérêt, attentif et cependant sans indulgence, porté par un Grec vaincu, otage à Rome même, à tout ce qui explique la puissance romaine. En 168 a.C. (et l'on peut tout aussi bien, sur ce point, se reporter au chapitre $\mathrm{V}$ des Considérations), Rome détient un pouvoir incontesté : Carthage, très longtemps grande puissance maritime, est alors doublement vaincue ; parmi les grandes monarchies hellénistiques, le royaume de Macédoine, après une première défaite de Philippe V à Cynoscéphales en 196, se trouve, après celle de son fils Persée à Pydna, cette même année 168, démembré en 167, partagé en quatre républiques autonomes 5 . Comme, dans l'intervalle, Rome a eu le dessus sur le roi de Syrie, Antiochos III, auprès de qui s'était réfugié Hannibal et à qui elle impose un traité sans indulgence en 188 à Apamée, il n'y a plus, en 168, aucune puissance assez forte pour se dresser contre elle : l'Égypte, en proie à de continuels conflits dynastiques, est mal gouvernée ; Pergame est un trop petit royaume. Quant à la Grèce, divisée, dans la guerre de Rome contre Persée, entre partisans et adversaires de Rome, elle est maintenue dans le calme par l'envoi en Italie d'otages soupçonnés d'hostilité ou de tiédeur envers Rome, tandis que les partis pro-romains sont partout portés au pouvoir (Histoires XXX, 13). D'ambassade achaïenne en ambassade achaïenne, toutes chargées de demander au sénat romain la libération des otages, Polybe passe ainsi dix-sept ans à Rome et, devenu le précepteur des enfants de Paul-Emile, de Scipion Emilien en particulier, il est en relations avec les

4 G. Benrekassa, Montesquieu, la liberté et l'histoire, Paris, 1987, p. 81. 
cercles dirigeants romains, rencontre les personnalités que Rome garde chez elle en otages, tel Démétrios, le futur souverain de Syrie, entend les commentaires sur la politique romaine. Il a aussi accès aux archives et peut voyager. Pour cet homme d'action et ce responsable politique, la compréhension des événements qui bouleversent son temps devient dès lors une autre forme d'action : il transforme l'histoire en leçon, expérience indispensable à quiconque se veut homme d'État.

Cet idéal de formation par l'histoire apparait dès le premier livre, au chapitre 35 : l'histoire des faits devient l'une des façons de se forger une expérience pour éviter de reproduire les erreurs du passé, mais sans danger pour le futur responsable politique qui peut, tout au contraire, méditer sur elle à loisir. Polybe érige cette idée en principe, reprend point par point la célèbre préface de Thucydide, en I, 22, 4 de l'Histoire de la

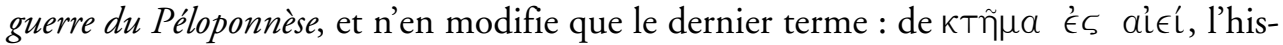
toire devient $\mu a ́ \theta \eta \mu a$ (III, 31, 12-13) ${ }^{6}$. Mais de même que Platon demande, pour le bien des États, des philosophes-rois ou des rois-philosophes, cette histoire instructive ne peut être écrite que par un historien qui, avec l'expérience de ce dont il parle, peut aller à l'essentiel et dégager la trame des faits (XII, 25g-h), voire assortir le récit des événements d'un "commentaire instructif » sur les comportements de ceux qui jouent un rôle dans l'action ( moins, pour former son destinataire, sur un improbable retour identique de l'événement que sur une expérience politique, acquise comme par procuration, et l'on connaît les réserves de Montesquieu sur une histoire qui se répéterait : " Les politiques ont beau étudier leur Tacite : ils n’y trouveront que des réflexions subtiles sur des faits qui auraient besoin de l'éternité du Monde pour revenir dans les mêmes circonstances 7 ." Enfin, cette histoire qui ne se limite pas au récit nu des événements, ne saurait non plus se borner à la seule histoire de Rome. Comme tout fait doit être examiné en fonction de ses causes et de ses conséquences et replacé dans un ensemble de faits synchroniques, l'ascension de Rome ne peut se comprendre qu'en fonction de causes internes à son propre développement et dans un contexte de causes plus général ; l'histoire doit donc se faire universelle et, à partir de $217(\mathrm{~V}, 105)$, toutes les évolutions particulières sont liées par l'historien qui les examine tour à tour, dans un ordre aussi constant que possible : affaires d'Italie, d'Espagne, de Grèce et d'Asie.

L'œuvre de Polybe, replaçant la conquête du pouvoir par Rome dans ce cadre international, est donc écrite à des fins d'histoire politique : pas plus que les Considérations, elle n'accorde aux faits historiques un intérêt ponctuel ; ce qui peut avoir valeur d'expérience, ce n'est pas tel ou tel fait ; c'est ce même fait qui, pris dans un ensemble plus général, établit une causalité analogue à celle que déterminent les lois physiques en définissant une évolution interne (formation, croissance et corruption),

6 Sur ce parallèle et, plus généralement sur le destinataire des Histoires, M. R. Guelfucci, "Des mots et des manières de lire : le lecteur de Polybe ", in Nomina rerum, Hommage à Jacqueline Manessy-Guitton, LAMA n ${ }^{\circ} 13$, 1994, p. 241-257.

7 Mes Pensées, III. Politique, 1764, éd. Gallimard, tome I, p. 1421. 
sous l'effet de causes aussi bien internes qu'externes (Histoires VI, 51 et 57). C'est dans cette perspective que, comme les Considérations, elle est une réflexion sur la nature du pouvoir, les conditions de sa conquête et de son maintien.

Dernière justification d'un parallèle qui ne se borne ni à la nature du sujet ni à la manière dont l'un et l'autre auteurs appréhendent celui-ci, Polybe est fréquemment et jusque dans une certaine partialité de ses jugements ${ }^{8}$ la source de Montesquieu, que les références faites à son œuvre renvoient au texte même ou au recueil d'extraits Sur les vertus et les vices que fit établir Constantin Porphyrogénète 9. Il semble que Montesquieu reconnaisse en lui non seulement l'historien qui garantit sa propre recherche ou le spécialiste des affaires et des techniques militaires, mais au détour de telle référence, l'historien politique, qu'il s'agisse de principes des gouvernements, d'étude du déclin d'un régime, des instruments plus ou moins adaptés de politique intérieure que peuvent être la crainte, la religion, le rôle des climats, l'équilibre des pouvoirs, sujets qui sont communs aux Considérations et à l'Esprit des lois.

\section{Les atouts de Rome dans la conquête de l'hégémonie}

Pour Polybe comme pour Montesquieu, Rome est une force conquérante, servie par les atouts que sont ses qualités militaires et ses institutions, rendus plus efficaces encore par des causes, externes cette fois : la faiblesse ou l'absence de sens politique des autres puissances, ce qui lui permet de développer un thème essentiel de sa propre propagande, la défense des peuples opprimés.

Ce sont d'abord des qualités militaires que les deux auteurs mettent en valeur, tout en montrant comment elles sont admirablement secondées par des moyens effectifs : Polybe consacre vingt-quatre chapitres du livre VI aux institutions militaires des Romains (ch. 19-42), étudiant non seulement l'armement des légionnaires, exposé auquel renvoie Montesquieu en note de son chapitre II, mais encore le camp romain, le service de garde, l'armée en marche. Tout le chapitre II des Considérations, inspiré de nombreux auteurs dont Polybe, est lui aussi consacré à "l'art de la guerre chez les Romains ". Ce que tous deux mettent surtout en valeur, ce sont l'organisation de l'armée, une tactique adaptée à toutes les situations parce qu'elle peut être diversifiée, la puissance de la légion se trouvant assistée de troupes légères et maniables, d'une cavalerie et de soldats formés à poursuivre l'ennemi : cette adaptation de l'armée au terrain apparaît ainsi comme l'un des éléments décisifs de la victoire des Romains à Cynoscéphales où, sur un terrain accidenté, la phalange de Philippe $V$ est défaite par

8 Voir en particulier la description sans nuance que fait Montesquieu des deux premières des trois confédérations grecques qu'il définit : Etoliens, Achaïens et Béotiens (Considérations, chapitre 5).

9 Sur la transmission du texte de Polybe, J. M. Moore, The manuscript tradition of Polybius, Cambridge, 1965. 
les manipules romains. Polybe, lui-même auteur d'un traité de tactique, l'explique longuement en XVIII, 29-30 des Histoires, et Montesquieu, observant au chapitre V le "bon sens ordinaire " de l'historien, souscrit à ce jugement. Mais Montesquieu indique aussi, au chapitre II, l'importance et le nombre des machines de guerre et, comme Polybe (VI, 50, 6), l'excellence de l'intendance.

Dans le chapitre II, Montesquieu marque aussi de manière appuyée l'extraordinaire capacité d'adaptation des Romains, leur manière de toujours tirer parti des avantages de l'ennemi, tout comme le fait Polybe avec l'exemple du bateau de guerre carthaginois pris, démonté et copié par les Romains, lors de la première guerre punique (Histoires I, 20, 15-16 et Considérations ch. IV) ${ }^{10}$. Un autre exemple, emprunté au livre I des Histoires (ch. 22), montre l'ingéniosité inventive du recours aux corbeaux grâce auxquels les Romains, transformant en combat terrestre une bataille navale ou, selon l'expression de Montesquieu (ch.II), « élud[dant] la science des pilotes », remportent la victoire de Myles.

Dans ce même chapitre II, Montesquieu montre aussi l'entraînement continuel et la discipline des soldats et résume de deux termes, en conclusion, l'ensemble de leurs qualités : la prudence et l'audace. Polybe, à la fin du livre I sur la première guerre punique, montre plus particulièrement la part de la $\psi v \chi 0 \mu a x i ́ a$ dans l'heureuse conclusion de la guerre pour Rome, l'« énergie de la dernière chance » dont les Romains firent preuve ${ }^{11}$, mais c'est précisément la réunion de ces deux qualités rares que Montesquieu met en avant, dosage idéal de réflexion et d'esprit d'entreprise qui, dans les Histoires, assure toutes les réussites.

Polybe et Montesquieu ont cependant trop de lucidité ou de réalisme pour imaginer que, si valeureux, bien armé et bien commandé que soit le soldat romain, il n'ait jamais besoin d'encouragements ou de garde-fous. Polybe explique donc, en VI, 37-39, comment les institutions militaires romaines prévoient récompenses et châtiments pour conforter la discipline et montre, au livre VI, comment l'amour de la gloire qui caractérise les Romains devient un avantage d'autant plus grand qu'il est renforcé par une

10 Sur le problème que pose le passage, utilisation ou non par Polybe d'un lieu commun loin de toute vérité historique, F. W. WALBANK, A Historical Commentary on Polybius, tome I, Oxford, 1957, p. 75-76.

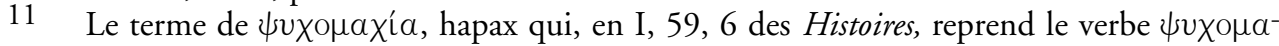
$\chi \in \tilde{\nu} \nu$ en $1,58,7$, pose un problème de sens et de traduction : il désigne moins un « combat désespéré " (expression ambiguë en français et seul sens que retient J. A. DE FOUCAULT, Recherches sur la langue et le style de Polybe, Paris, 1972, p. 389) que l'acharnement mis dans un combat à mort qui, au prix de l'engagement des fortunes privées des Romains pour sauver l'État, assure à Rome la victoire. Ce peut être, pour garder la valeur positive du terme, l'énergie du désespoir, l'énergie de la dernière chance qui sauvent la situation. Polybe crée le substantif à partir de la comparaison avec des « coqs de race qui luttent pour

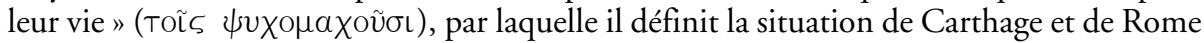
(Histoires, I, 58, 7-9). 
véritable émulation : les jeunes Romains sont encouragés par la promesse de distinctions (VI, 39, 1-11) et par la cérémonie des imagines (VI, 52, 11-53). Mais les châtiments, décrits par Polybe (VI, 37-38), rappelés en partie par Montesquieu (XVIII), sont autant dissuasifs. Les deux auteurs montrent aussi comment, après la victoire et pour éviter toute dérive, le pillage est très soigneusement codifié (Histoires X, 16 et Considérations I) et tous deux font apparaître l'intérêt du serment pour la discipline des armées (Histoires VI, 33 et Considérations ch.1).

Le serment, dont Montesquieu dit qu'il « fut toujours le nerf de leur discipline militaire " (I), et la religion, de façon plus générale, jouent en effet un rôle essentiel, pour Polybe et pour lui, dans la vie des soldats comme de l'ensemble des citoyens romains : ni l'un ni l'autre ne semblent considérer la religion romaine pour ce qu'elle fut vraiment ${ }^{12}$, mais la jugent plutôt, dans la ligne des sophistes du ve siècle classique, comme une façon de garantir les lois humaines par une crainte irrationnelle. Montesquieu, reconnaissant au chapitre $\mathrm{X}$ des Considérations, le rôle de la pietas romaine, se montre toutefois beaucoup plus nuancé et suit moins littéralement Polybe que dans sa Dissertation sur la politique des Romains dans la religion, lue en 1716 à l'Académie de Bordeaux et imprimée après la mort de l'auteur. Dans ce traité de jeunesse, Montesquieu résume, mais sans nuance aucune, la thèse que soutient Polybe, en VI, 56 des Histoires:

Polybe met la superstition au rang des avantages que le peuple romain avait par-dessus les autres peuples : ce qui paraît ridicule aux sages est nécessaire pour les sots; et ce peuple qui se met si facilement en colère, a besoin d'être arrêté par une puissance invincible.

Polybe oppose en fait à une très hypothétique cité de sages, une société humaine où les passions peuvent se donner libre cours et montre comment celles-ci peuvent être canalisées par une $\delta \epsilon \iota \sigma \iota \delta \iota \mu \nu v i ́ \alpha$, non pas "superstition ", sens qui est celui du terme dans les textes de Théophraste ou, après Polybe, de Plutarque, mais littéralement une " crainte des dieux ". Celle-ci retient non seulement le peuple, mais jusqu'aux magistrats romains, comme la crainte de voir ses prérogatives limitées empêche chacun des trois corps qui composent l'État d'empiéter sur celles des deux autres.

Car c'est aussi l'équilibre de la constitution romaine, composée de trois pouvoirs, le sénat, les consuls, le peuple, qui est, au terme d'une longue expérience selon Polybe (Histoires VI, 10, 13-14), « depuis sa naissance " d'après Montesquieu (VIII), la sauvegarde du pouvoir romain. Parce qu'aucun des trois corps ne peut prédominer sur les autres, la constitution est un frein à l'autorité arbitraire d'un seul corps, à la corruption

12 Pour les idées de Polybe sur la religion romaine, J. de RomilLY, " L'historiographie grecque ", in Actes du IXe Congrès de l'Association G. Budé, Rome, 13-18 avril 1973, p. 129, et H. DÖRrIE, "Polybios über Pietas, Religio und Fides ”, in Mélanges Boyancé, Rome, 1974, p. 251-272. 
des mœurs. Polybe définit le jeu de la tripartition du pouvoir à Rome, en VI,11, 1118, 8. Montesquieu n'y revient pas et s'en explique :

On souhaiterait peut-être que j'entrasse ici dans le détail du gouvernement politique de la République romaine ; mais je renverrai à Polybe, qui a admirablement bien expliqué quelle part les consuls, le Sénat, le Peuple, prenaient dans ce gouvernement ; d'autant mieux qu'il parle d'un temps où la République venait d'échapper à de si grands périls et faisait actuellement de si grandes choses 13 .

Polybe et Montesquieu montrent néanmoins qu'aucune puissance n'est définitive, que la décadence est au contraire inéluctable, par comparaison avec d'autres régimes analogues, Carthage essentiellement. Pour Polybe, qui reprend le schéma de décadence platonicien de la République pour le styliser et le modifier à la manière d'Aristote (Pol. III,7), il n'y a pas de décadence continue vers ce qui serait le pire régime : la tyrannie, mais il existe trois formes de bons gouvernements (royauté, aristocratie, démocratie) qui évoluent vers leur forme corrompue (tyrannie, oligarchie, ochlocratie) ; Polybe précise l'agent de la corruption, la $\pi \lambda \epsilon \mathrm{O} \in \xi\lfloor\alpha$, désir inhérent à la nature humaine d'avoir, sans effort et sans contrepartie, toujours plus. Avant le premier régime règne une société sans loi où domine le droit du plus fort ; au terme de l'ochlocratie, le peuple, trompé par un démagogue, lui remet toute autorité et c'est à nouveau, comme au commencement, un monde sans loi ${ }^{14}$. La constitution romaine, comme celle de Sparte ou de Carthage, pallie ces risques d'évolution en équilibrant, dans un même gouvernement, trois formes positives de régime : royauté avec les consuls, aristocratie avec le sénat, démocratie avec le pouvoir de contrôle donné au peuple. Néanmoins, en période de trop grande abondance, les tensions s'accentuent, les passions, quoique bridées par la tripartition des pouvoirs, s'enflent jusqu'à ne plus pouvoir être canalisées et c'est toujours le peuple qui est à l'origine de la rupture d'équilibre. Exemple de cette théorie générale, la défaite de Carthage devant Rome, dans la deuxième guerre punique, s'explique ainsi (VI, 51, 3-7).

Historiquement, l'analyse n'est pas fausse : les Barcides et plus particulièrement Hannibal apparaissent comme soutenus par le parti populaire tandis que les autorités carthaginoises sont plus réticentes. Montesquieu adopte très nettement l'explication de Polybe : "Carthage, devenue riche plus tôt que Rome, avait aussi été plus tôt corrompue " (début du chapitre IV) : "Ce furent les conquêtes mêmes d'Annibal qui commencèrent à changer la fortune de cette guerre. Il n'avait pas été envoyé en Italie

13 “Dossier des Considérations", in Montesquieu, Euves complètes, éd. Gallimard, 1951, tome II, p. 216-217, n 158, sous la rubrique : "155-159. - Cela n'a pu entrer dans les Romains ".

14 Sur la hiérarchie des formes de régime, M.-R. GuELfuCCI, "Les origines de la société politique d'après un historien : Polybe (Histoires 6, 5 sq.) ”, in J.-M. GALY et A. THIVEL éd., Les origines de l'homme, Nice, 1998, p. 153-169. 
par les magistrats de Carthage ; il recevait très peu de secours, soit par la jalousie d'un parti, soit par la trop grande confiance de l'autre. " Hannibal ne peut donc compter, en terre étrangère, que sur lui-même. Tous deux, en admirant le génie de l'homme ${ }^{15}$, donnent donc sa défaite comme attendue, dans l'ordre d'évolution de l'histoire (Histoires XV, 16-17 et Considérations V).

Mais la $\pi \lambda \epsilon O \nu \in \xi$ ía mine aussi le pouvoir extérieur des États. Ainsi la puissance de Rome, à son apogée au moment de la seconde guerre punique, tient non seulement à la valeur de ses citoyens et à ses institutions, mais aussi aux défauts politiques des puissances qui auraient pu lui tenir tête : Montesquieu, aux chapitres IV (à propos de Carthage) et $\mathrm{V}$ des Considérations, fait la synthèse d'éléments qui apparaissent aussi chez Polybe, mais plus disséminés dans leur ordre chronologique. Nous n'en retiendrons qu'un, qui concerne deux étapes majeures dans la conquête romaine et qui permet aux Romains de faire figure de libérateurs : la méconnaissance de l'intérêt politique à long terme qui permet, seul, qu'en exerçant le pouvoir avec la justice nécessaire ou, au pire, en ne se montrant bienveillant ou clément envers les populations sujettes ou ennemies qu'au nom du simple intérêt, on gouverne sans révolte de leur part, voire en se gagnant leur reconnaissance. Lidée est au cœur de la pensée politique de Polybe et elle est reprise par Montesquieu. Polybe, au livre I (71-72), donne l'exemple de Carthage : alors même qu'elle doit faire face à la révolte de ses mercenaires, elle est trahie par les populations africaines soumises à son autorité à cause de sa politique tyrannique et de ses exactions. Polybe voit là un aboutissement très logique : "Tout cela prouve qu'un bon gouvernement doit toujours prendre ses décisions non seulement en tenant compte du présent, mais aussi, et plus encore, en pensant à l'avenir » $(72,7)$. Mais sans aucunement tirer la leçon des événements, Carthage reproduit la même politique en Espagne, au moment de la guerre contre Scipion, adoptant à l'égard des populations indigènes une politique d'oppresseur, tandis que Scipion se fait, de façon presque théâtrale si l'on en croit le

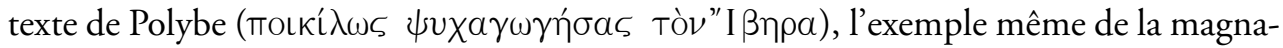
nimité et de la clémence (X, 34-35 et 35.2 tout particulièrement). Les Espagnols prennent donc le parti des Romains. Montesquieu, qui se réfere à un texte de Polybe encore antérieur (Histoires IX, 11), conclut, au chapitre IV, sur cette imprévoyante attitude des Carthaginois :

Et, si l'on fait attention aux sommes immenses qu'il leur en coûta pour soutenir une guerre où ils succombèrent, on verra bien que l'injustice est mauvaise ménagère, et qu'elle ne remplit pas même ses vues.

15 Montesquieu, Considérations IV : "Quand on examine cette foule d'obstacles qui se présentèrent devant Annibal, et que cet homme extraordinaire surmonta tous, on a le plus beau spectacle que nous ait fourni l'antiquité. " Pour Polybe : "Il y en a bien peu... qui soient également capables de se servir de la fortune, et de l'attendre ; et qui, avec cette disposition d'esprit qui donne de la méfiance avant que d'entreprendre, aient celle de ne craindre plus rien après avoir entrepris. » 
Polybe, commentant les mêmes événements, les assortit, en X, 36, d'une règle politique, écho à la condamnation des Carthaginois au livre I et véritable mise en garde pour tout pouvoir établi et trop sûr de lui :

Les Carthaginois n'eurent ainsi que ce qu'ils méritaient. Ils avaient cru qu'il existait une méthode pour acquérir l'empire et une autre pour le garder. Ils n’avaient pas compté que ceux qui préservent le mieux leur suprématie sont ceux qui restent le plus fermement attachés aux maximes qui leur ont permis de l'instaurer.

Mais Rome non plus n'est pas à l'abri et le risque, accru par l'obséquiosité des rois vaincus, la possible transformation d'un régime mesuré en hégémonie autoritaire et le luxe de la conquête, devient paradoxalement d'autant plus grand que sa puissance paraît incontestée.

\section{Les risques d'un pouvoir incontesté. Éléments de philosophie politique et de philosophie de l'histoire}

Montesquieu réprouve l'attitude de certains alliés, obséquieuse parfois jusqu'à en être servile, au chapitre VI des Considérations:

Quoique le titre de leur allié fût une espèce de servitude, il était néanmoins très recherché ; car on était sûr qu'on ne recevait d'injures que d'eux, et l'on avait sujet d'espérer qu'elles seraient moindres : ainsi, il n'y avait point de services que les peuples et les rois ne fussent prêts de rendre, ni de bassesses qu'ils ne fissent pour l'obtenir.

Polybe condamne, dès avant l'hégémonie de Rome, l'Achaïen Callicratès qui, dès son ambassade à Rome en 180 a.C, est, selon lui, l'initiateur de cette attitude (XXIV, 8,$6 ; 9,2 ; 10,4$ et 5) 16 et, aux lendemains de Pydna, le roi de Bithynie Prusias, au comportement "tout à fait indigne de la majesté royale" $(\mathrm{XXX}, 18,2)$. La fin du

16 F. W. Walbank, Comm., t. III, 1979, p. 263. J.-L. FerRary, Philhellénisme et impérialisme, aspects idéologiques de la conquête romaine du monde hellénistique, École française de Rome, 1988, résume (p. 300-301) le scepticisme généralement adopté à l'égard du texte de Polybe, mais lui-même reprend l'étude du passage (p. 299-306) pour en faire clairement apparaître le double aspect en montrant comment Polybe, après 168, met l'accent sur le changement d'attitude du sénat. Pour une explication de l'attitude de Callicratès, qui aurait compris que l'alliance romaine de 192 n'équivalait pas à la toute-puissance de la Confédération, mais plutôt à un rapport de clientèle qui permettrait une détente des relations avec Rome, voir E. WILL, Histoire politique du monde hellénistique, t. II, p. 244-245, qui donne les références aux thèses qui vont dans ce sens; P. PÉDECH, enfin, fait état, pour comprendre Callicratès, de la politique de raidissement de Rome envers les Grecs : $L a$ méthode historique de Polybe, Paris, 1964, note 196, p. 295. 
passage montre le danger majeur de l'attitude de Prusias qui, en supprimant la possibilité ménagée à une discussion constructive, fausse le jeu politique en lui substituant l'acceptation inconditionnelle de la force : «S'étant ainsi conduit de façon absolument méprisable, il obtint du reste, et justement pour cette raison, une réponse amicale. » De même que les institutions sont durables quand les pouvoirs s'équilibrent, de même les relations internationales entre puissances équivalentes ou entre puissances alliées ne peuvent, selon Polybe, reposer que sur un dialogue qui continue à équilibrer, par l'isologia, des forces qui peuvent, elles, être disproportionnées ; de cette manière, l'évolution vers une forme d'hégémonie corrompue, tyrannique, peut être évitée. Cette règle est fondamentale dans les Histoires (I, 83, 2-4 ; XXIV, 13-15).

Or, dans le jeu des fragments que nous possédons, Polybe souligne la politique du Sénat à l'égard des souverains ou des peuples qui pourraient se révéler des rivaux, tel Démétrios gardé en otage à Rome quinze ans durant. Car, malgré l'injustice de sa détention (XXXI, 2, 1) ${ }^{17}$ et la sympathie des sénateurs à son égard, le Sénat, en tant que corps constitué cette fois, refuse de le laisser repartir en Syrie pour accéder au trône :

Il éprouvait, je pense, quelque inquiétude en songeant que Démétrios était dans la force de l'âge et il jugeait plus favorable à ses intérêts propres, la présence sur le trône d'un prince encore tout jeune et incapable d'agir ; la suite des événements le fit bien voir (XXXI, 2, 7-8).

Démétrios, contre l'avis de Polybe, plus lucide, présente deux fois sa requête avec le même insuccès (XXXI, 11, 11-12) et Polybe, probablement sûr de l'appui des Scipions ${ }^{18}$, l'aide à s'enfuir (XXXI, 12, 7-15).

C'est aussi la duplicité d'un sénat dissimulateur, attendant l'occasion et le bon prétexte pour faire une guerre, garantissant ses traités en intimidant ses nouveaux alliés par le maintien d'otages à Rome, intervenant dans les affaires intérieures, ayant pour politique de diviser pour mieux régner, que dénonce Montesquieu dans tout le début du chapitre VI des Considérations : "Mais surtout leur maxime constante fut de diviser. " Dans les Histoires, cette pratique apparaît dès qu'il peut y avoir rivalité entre deux frères pour le pouvoir, avant 168 déjà dans le cas de Démétrios et de Persée, puis à l'égard d'Attale et d'Eumène ou des deux Ptolémées (XXXI, 10 et 17-19; XXXIII, 11). Dans le cas d'Eumène et d'Attale, la manœuvre échoue (XXX, 3 ; XXXI, 1 ; 32), Eumène II ayant dépêché auprès d'Attale son médecin Stratios qui contrecarre la

17 Il garantissait à Rome la loyauté de son père Séleucos IV ; mais son père assassiné et son oncle Antiochos IV ayant usurpé le pouvoir, sa détention n'avait plus lieu d'être. A la mort de celui-ci, il est le prétendant légitime au trône de Syrie tandis que le fils d'Antiochos IV n'est qu'un enfant et que Lysias exerce la régence.

F. W. Walbank, Comm. III, p. 478 ; sur la prise de position officielle des sénateurs, leur opinion privée et leur attitude au moment de la fuite du prince, E. WiLl, Histoire politique du monde hellénistique, II, p. 366. 
mauvaise influence des Romains (XXX, 2). Mais le sénat reproduit la même politique, diviser tout en privilégiant ses propres intérêts, à l'égard d'États rivaux et favorise par exemple, malgré l'avis du consul, deux des peuples celtibères contre le troisième (XXXV, 3, 4), Massinissa contre Carthage (XXXI, 21, 5-8), " non que les Carthaginois fussent dans leur tort mais parce que leurs juges étaient persuadés qu'il était de leur intérêt ( $\sigma u \mu \phi \epsilon ́ \rho \in \nu \nu \sigma \phi i ́ \sigma)$, de se prononcer contre eux » (\$6). Le changement est tout particulièrement commenté à propos des affaires d'Égypte (XXXI, 10, 7), avec la distinction entre une attitude qui serait véritablement politique (définie par l'expression

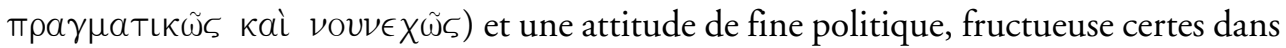
l'immédiat, mais pernicieuse à plus ou moins long terme pour ceux qui la pratiquent

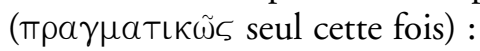

Telle est en effet aujourd'hui la politique ordinaire des Romains : ils tirent habilement

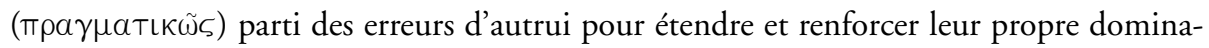
tion et, ce faisant, ils gagnent la reconnaissance de ceux qui commettent des fautes, en

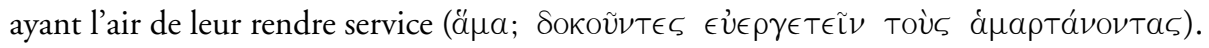

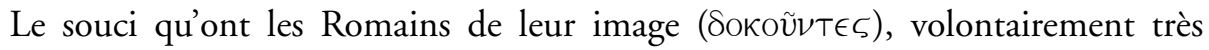
marqué dans les Histoires, n'est pourtant pas entièrement négatif tant qu'il garantit le respect des formes du droit. Ainsi Polybe note dans toutes ses nuances l'embarras des sénateurs, à l'occasion de la visite d'un roi qu'ils ne veulent pas recevoir (XXX, 1 ou XXX, 19, 2) ; de la requête des Achaïens qui viennent leur demander de juger les otages ou de s'en remettre à eux pour le faire (XXX, 32, 6), de l'arrivée à Rome des coupables que Démétrios Ier leur envoie de Syrie (XXXII, 2, 2). Mais le Sénat ne veut surtout ni se déconsidérer aux yeux de l'étranger ni négliger les intérêts romains. Or dans le cas des Achaïens (XXX, 32, 7), le choix d'une solution met en balance la justice (il ne lui appartient pas de juger les otages) et les intérêts de Rome (il ne peut avoir l'air de favoriser des opposants à ses propres alliés). Dans les trois cas, sa réponse est donc un compromis et dans le dernier, par exemple, le Sénat garde le présent, mais n’accepte pas les prisonniers et adresse au roi une réponse qui, avec le vague des formules officielles ${ }^{19}$, ne définit pas ce qu'elle attend de lui (XXXIII, 3, 13) : "C'est donc tout juste s'il consentit à recevoir les ambassadeurs, car il tenait à garder ses griefs intacts, de façon à pouvoir, quand il le voudrait, se poser en accusateur » (XXXII, 3, 11-12). Mais le cas le plus clair de duplicité que commente Montesquieu au chapitre VI des Considérations, en renvoyant explicitement à Polybe (XXXII, 13, 1-4 et 9), est cependant l'attitude du Sénat à l'égard des Dalmates : "Leur coutume étant de parler toujours en maîtres, les ambassadeurs qu'ils envoyoient chez les peuples qui n'avoient point encore senti leur puissance, étoient sûrement maltraités : ce qui étoit un prétexte sûr pour faire une nouvelle guerre."

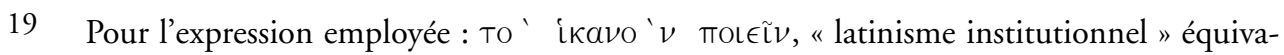
lant à satisfacere, M. Dubuisson, Le latin de Polybe, Paris, 1985, p. 173-176. 
La politique romaine a donc le double caractère de toujours respecter les apparences du droit, mais d'être d'abord fondée sur le calcul de l'intérêt romain. L'on est donc en droit de se demander si, après avoir su conquérir l'hégémonie parce qu'elle était la cité la plus apte à la mériter, Rome n'est pas, après 168, engagée dans un processus de décadence et si elle est capable d'exercer le pouvoir suprême après la victoire (III, 4). Dans le cas de Rome, la réponse est d'autant moins facile que Polybe lui-même ne tranche pas et fait toujours apparaître, même dans la déclaration de guerre contre Carthage (XXXVI, 2), le respect des formes, la recherche d'une " occasion favorable " et d'un "prétexte honorable " à côté de la poursuite exclusive des intérêts de la cité. Ainsi, là où Montesquieu (ch. VI) montre clairement le cynisme des Romains, Polybe laisse certes entrevoir la lente corruption des rapports internationaux, mais n'en retient pas moins, d'abord, la subsistance d'une certaine légalité, susceptible, si les principaux dirigeants romains corrigent leurs erreurs, de rendre sa force à l'hégémonie romaine. C'est toute l'ambiguïté de sa position au moment où Rome décide de détruire Carthage. Car ce qu'il veut noter, en rupture avec une absence de droit des gens dont l'exemple le plus significatif des Histoires, bien qu'il ne soit pas dénué de partialité, est l'exemple étolien, c'est l'existence à Rome d'un droit de la guerre : celle-ci doit être déclarée et les Romains se sentent au moins tenus, devant l'opinion internationale, de la justifier ${ }^{20}$.

Pour Rome, le risque politique est donc double : à l'extérieur, celui de la marche à l'empire, impérialisme qui, selon la maxime de Périclès en son temps ${ }^{21}$, ne peut engendrer que la haine et la révolte, et parallèlement, à l'intérieur, les conséquences, confortables mais pernicieuses, de la conquête 22 et, avec elles, l'apparition de chefs du peuple, rompant le fragile équilibre constitutionnel. Montesquieu, informé de la suite des événements, reprend en l'inscrivant dans le temps l'explication théorique de l'anacyclose (Hist., VI, 57). Il fait ainsi apparaître, au livre XI de l'Esprit des lois, un premier

20 Sur cette attitude constante du sénat, nous renvoyons à " Guerres et diplomatie romaines dans les Histoires de Polybe ", à paraître in Guerres et diplomatie romaines (IVe-IIIe siècles), E. Caire et S. Pittia éd., Aix-en-Provence, Actes du colloque international tenu en 2005. Thucydide, Histoire de la guerre du Péloponnèse, II, 63, 2 ; cf. aussi III, 40, 4.

22 Pour Polybe, à la fin de l'exposé de la première guerre punique, comme pour Montesquieu qui cite Tite-Live (Considérations III), Rome ne serait plus capable de ses réussites d'autrefois, la cupidité et la corruption (Considérations VI) dénaturant même les serments. Montesquieu, sans remarquer que Polybe lui-même montre une évolution négative dès son époque, à partir des guerres au-delà des mers (Histoires VI, 56, 12-15 et XVIII, 35, 12), oppose, au chapitre X, l'époque de Cicéron à celle de Polybe. Pour Polybe comme pour lui, Rome court le risque d'oublier ce qui a fait sa force, mais alors que l'historien grec dénonce jusqu'à l'oubli des vertus militaires (Histoires XXXV, 4), Montesquieu, peut-être parce qu'il traite moins d'une période particulière que de l'histoire romaine en général, fait encore l'éloge des valeurs guerrières de Rome, exceptionnellement conservées " au milieu des richesses, de la mollesse et de la volupté" (X). 
facteur de déséquilibre des forces politiques avec la possibilité donnée au peuple, déjà doté du droit de veto de ses tribuns, de s'opposer au sénat (ch. 6) ; au chapitre IX des Considérations, il montre comment l'armée se met, dans un empire devenu trop grand pour la cité, à choisir elle-même comme chefs ses généraux et à leur donner tout pouvoir :

Tandis que le peuple de Rome ne fut corrompu que par ses tribuns, à qui il ne pouvait accorder que sa puissance même, le sénat put aisément se défendre, parce qu'il agissait constamment ; au lieu que la populace passait sans cesse de l'extrémité de la fougue à l'extrémité de la faiblesse. Mais quand le peuple put donner à ses favoris une formidable autorité au dehors, toute la sagesse du sénat devint inutile, et la république fut perdue.

Pour l'un comme pour l'autre, la décadence, sous l'effet conjugué d'une vertu civique devenue moindre et d'un état de la constitution où le parti populaire se refuse à la discipline nécessaire, est donc inéluctable et c'est même, pour Polybe, une loi physique (VI, 57, 1-2). L'influence du texte polybien marque jusqu'au jugement de Montesquieu sur le régime anglais qu'il donne en modèle à son tour : "Rome, Lacédémone et Carthage ont bien péri. Il périra lorsque la puissance législative sera plus corrompue que l'exécutrice " (Esprit des lois, XI, 6).

Cependant, c'est dans la logique même du double processus d'une diminution de la force de l'État que réside la possibilité pour tout pouvoir de freiner sa propre déchéance. Car si le processus de déchéance est naturel, il est par là même pour Polybe (Histoires VI, 9, 10-14) explicable et prévisible, et l'on peut, quand on en comprend le fonctionnement, juger de l'état d'une évolution (VI, 9, 11-13 ; VI, 57, 3-4), et donc corriger celle-ci. Quant à Montesquieu, à une conception d'un monde gouverné par la fortune, il oppose la recherche des causes profondes qui commandent les événements, "l'allure principale » entraînant les " accidents particuliers " (Considérations XVIII). Ils trouvent donc tous deux au problème politique un remède politique, maintenir l'équilibre des pouvoirs.

Tous deux admettent, en effet, que l'équilibre politique doit compter avec les divisions et les intérêts contraires qui traversent le corps politique et ne peut donc être que paradoxal (Histoires VI, 18 et 18, 7-8 en particulier, Considérations IX). À la fin du chapitre IX des Considérations, Montesquieu, réfléchissant sur l'évolution des grandes puissances que sont Rome, Carthage, Athènes, les républiques italiennes, l'Angleterre, donne une loi générale qui exige l'effort : "En un mot, un gouvernement libre, c'est-àdire toujours agité, ne sauroit se maintenir, sill n'est, par ses propres lois, capable de correction. » Plus important, c'est même dans ce jeu des forces et des structures de l'État qu'il voit le signe de la vraie liberté politique, harmonisation de toutes les dissonances (Considérations IX) :

On n'entend parler, dans les auteurs, que des divisions qui perdirent Rome ; mais on ne voit pas que ces divisions y étoient nécessaires, qu'elles y avoient toujours été et qu’elles y devoient toujours être. Ce fut uniquement la grandeur de la république qui fit le mal, 
et qui changea en guerres civiles les tumultes populaires. Il falloit bien qu'il y eût à Rome des divisions : et ces guerriers si fiers, si audacieux, si terribles au dehors, ne pouvoient pas être bien modérés au dedans. Demander, dans un État libre, des gens hardis dans la guerre, et timides dans la paix, c'est vouloir des choses impossibles : et, pour règle générale, toutes les fois qu'on verra tout le monde tranquille dans un État qui se donne le nom de république, on peut être assuré que la liberté n'y est pas.

Ce qu'on appelle union dans un corps politique, est une chose très équivoque : la vraie union est une union d'harmonie, qui fait que toutes les parties, quelque opposées qu'elles nous paroissent, concourent au bien général de la société ; comme les dissonances, dans la musique, concourent à l'accord total. Il peut y avoir de l'union dans un État où l'on ne croit voir que du trouble ; c'est-à-dire une harmonie d'où résulte le bonheur, qui seul est la vraie paix. Il en est comme des parties de cet univers, éternellement liées par l'action des unes, et la réaction des autres.

Mais pour montrer la difficulté d'un tel résultat, Montesquieu recourt, en V, 14 de l'Esprit des lois, à la même métaphore des poids et contrepoids qu'emploie Polybe en VI, 10, 7 ; 10, $10 ; 14,3$ des Histoires:

Pour former un gouvernement modéré, il faut combiner les puissances, les régler, les tempérer, les faire agir ; donner, pour ainsi dire, un lest à l'une, pour la mettre en état de résister à une autre ; c'est un chef-d'œuvre de législation, que le hasard fait rarement, et que rarement on laisse faire à la prudence. Un gouvernement despotique, au contraire, saute pour ainsi dire, aux yeux ; il est uniforme partout : comme il ne faut que des passions pour l'établir, tout le monde est bon pour cela.

Cette politique modérée n'est donc en aucun cas un idéal frileux de médiocrité, mais bien au contraire, chez Polybe comme chez Montesquieu, la perception courageuse et très lucide de la fragilité de l'équilibre politique, véritable résultante de forces contraires qui se neutralisent ou, au sens étymologique et latin du terme, compensation des pouvoirs, faute de quoi la raison et les lois finissent par céder le pas à la force.

\begin{tabular}{l} 
MARIE-ROSE GUELFUCCI \\
\hline Université de Franche-Comté \\
ISTA (EA 4011) \\
UFR Sciences du Langage, de l'Homme et de la Société \\
30-32 rue Mégevand \\
25030 Besançon Cedex \\
marie-rose.guelfucci@wanadoo.fr
\end{tabular}

